\title{
Effects of Magnetic Stimuli on Vigilance: A Heart Rate Variability Analysis
}

Huanhuan Cui ${ }^{1}$, Miao Tian ${ }^{1}$, Yufeng Ke ${ }^{1}$, Jinwen $\mathrm{Wei}^{1}$, Xuemin Wang ${ }^{1}$, Hongzhi Qi ${ }^{1}$, Dong Ming ${ }^{1}$, Robert Allan Backer ${ }^{2 *}$, Peng Zhou ${ }^{1 *}$

\footnotetext{
${ }^{1}$ Department of Biomedical Engineering Institute, Precision Instruments and Opto-Electronics Engineering, Tianjin University, Tianjin 300072.

${ }^{2}$ University of Delaware, 1075A McKinly Lab, 63 E Delaware Ave, Newark, DE USA 19717.
}

*Corresponding to: Robert Backer, University of Delaware, 1075A McKinly Lab, 63 E Delaware Ave, Newark, DE USA 19717, E-mail: backer@udel.edu; Peng Zhou, Precision Instruments and OptoElectronics Engineering, Tianjin University, Tianjin 300072, China, E-mail: zpzpa@vip.sina.com.

\begin{abstract}
Recent advances have demonstrated that magnetic stimulation provides an effective and noninvasive way to enhance neurological functioning across multiple cognitive and motor domains. The present study extends this work by investigating the potential of magnetic fields to enhance human vigilance, measured via heart rate variability (HRV). In a sham-controlled study, HRV signal was analyzed across both the time and frequency domains for 15 participants as they were exposed to magnetic stimulation. Our findings demonstrate that magnetic stimulation is capable of fortifying vigilance, as indexed by several key HRV components: increased RR variability, decreased rootmean-square of successive difference-value (RMSSD), reduced low frequency (LF) signal power, and increased high frequency (HF) signal power. Given the indication that magnetic stimuli are indeed capable of improving these key correlates of vigilance, we discuss several important implications.
\end{abstract}

Keywords: Heart Rate Variability; Magnetic Stimuli; Vigilance

\section{INTRODUCTION}

Vigilance, defined as the ability to maintain concentrated attention over prolonged periods of time ${ }^{[1]}$ is an important construct that is integral for assessing performance across a wide variety of functional contexts. 
An important component to assess when conducting research in this area is vigilance decrement-gradual deterioration in performance efficiency during vigilance tasks ${ }^{[2]}$, characterized by "mindlessness", or a withdrawal of attention from the monitoring assignment. Vigilance decrement is a frequently observed phenomenon during vigilance paradigms ${ }^{[3-6]}$. Recent work has focused on understanding the implications of HRV. HRV is a key marker of both stress and one's performance, specifically, the ability to remain vigilant ${ }^{[7]}$.

The vigilance decrement process is intimately connected to changes in the autonomic nerves system (ANS), which is susceptible to negative influences from one's environment (e.g., when the demands of personal or professional obligations exceed one's resources to meet necessary requirements). More specifically, ANS is subdivided into two systems ${ }^{[8]}$, which can be conceptualized as competing with one another as the ANS attempts to regulate processes in the body in response to demands from the environment: The parasympathetic nervous system is responsible for mediating neurobiological stress responses, regulating them when the body is not immediately responding to challenges (i.e. the "fight-or-flight" response) ${ }^{[9]}$. The sympathetic nervous system, contrastingly, initiates stress responses necessary for mobilizing the body to respond to immediate challenges (e.g., releasing epinephrine and norepinephrine, constricting blood vessels, and restricting blood supply to vital organs). Changes from individuals' baseline balance of sympathetic-parasympathetic activity can indicate the normal or fatigue state of ANS ${ }^{[10]}$.

A well-established, noninvasive measure of vigilance decrement is the electrocardiogram (ECG), which records the heartbeat ${ }^{[7]}$. ECG indicates the normalcy of one's heart rhythm, and it is a staple component of comprehensive physical exams. Specifically, an important measure that can be derived from ECG is heart rate variability (HRV), which describes the amount of variation in the time interval between two given heartbeats. HRV can also be referred to as RR variability, where each "R" is a peak in the ECG signal, indicating a heartbeat, and thus RR is the interval from one beat to the next. HRV can reveal patterns indicative of drowsiness and fatigue, and it is therefore able to provide information gauging mental states, such as level of vigilance.

Specifically, research has established a connection between decreased HRV and increased sympathetic nervous system activity (relative to decreased parasympathetic activity), which coincides with improved vigilance ${ }^{[11]}$. HRV is examined via two dimensions: the frequency and time domains. Frequency domain signal is decomposed into specific frequency bands, after which the number of RRs is measured within each band. Conventionally, the signal is partitioned into low frequency (LF; $0.04-0.15)$ and high frequency (HF; $0.15-0.4$ $\mathrm{Hz}$ ). Decreased HRV is instantiated by reduced LF power and increased HF power. In the time domain, decreased HRV is reflected in greater mean RR and greater root-mean-square of successive differencevalue (RMSSD) ${ }^{[12]}$. RMSSD captures high-frequency periodic fluctuations in heart rhythm, and therefore is appropriate for indexing fast fluctuations between sympathetic and parasympathetic activity, or vagal control. (Refer to Methods sections D and E for a more detailed description of these indexes.) In this study, we supplement our HRV measures with a behavioral assessment of vigilance: the psychomotor vigilance task (PVT) ${ }^{[13]}$. PVT, a reaction-time measure of how quickly subjects respond to a visual stimulus, is a reliable and widely-used index of vigilance degradation $^{[14]}$.

One promising way to ameliorate the process of vigilance decrement, as gauged by HRV measures, is by applying magnetic stimulation to the brain. Studies have shown that magnetic stimulation can regulate a range of psychological processes, from cognitive functioning to motor behavior ${ }^{[15-16]}$. In particular, low frequency-pulsed magnetic stimulation has been widely used in clinical settings because of it is safe, noninvasive, and painless, among other advantages. Notably, Yasaman Zandi Mehran et al. found that, by applying a $45 \mathrm{~Hz}$, low frequencypulsed magnetic field $(360 \mu \mathrm{T})$ to a central portion of the cortex $(\mathrm{Cz})$, they were able to enhance levels of attention ${ }^{[17]}$. This study builds on the understanding that magnetic stimulation can induce changes in the brain's electrical rhythms, which are capable of enhancing mechanisms relevant to vigilance. Further, Lichen Shi et al. determined that one earlyprocessing area highly correlated with vigilance to visual stimuli was an occipital cortex region near the posterior area of the scalp ${ }^{[18]}$. Therefore, we apply a novel magnetic stimulation device to stimulate this occipital region, in order to explore how stimulation may intervene to assist with vigilance.

This study manipulates vigilance degradation through an N-back working memory task, which requires participants to recall complex sequences of visual stimuli and thus produces progressive cognitive load that eventually results in performance decline. N-back work memory task induced mental fatigue, and thus reduced the vigilance. PVT is used to detect this decline in cognitive performance, that is the decline in vigilance. Under the conditions of the $\mathrm{N}$-back paradigm, we demonstrate the effectiveness of magnetic stimulation, to attenuate the process of vigilance degradation. 


\section{METHODS}

\subsection{Subjects}

A total of 15 healthy subjects ( 8 men, mean age 25 \pm 3 years; all right-handed) participated in the study. All subjects were right-handed in order to control the variables. For 24 hours prior to the experiment, subjects were instructed to abstain from caffeine and alcohol, as well as to obtain adequate sleep (a full 8 hours), to establish a normative baseline state for their participation. Experiments were performed in an electrophysiological laboratory, equipped with sound insulation and electrical shielding materials. A consistent room temperature was maintained throughout the experiment. All subjects gave informed consent after the nature and possible consequences of the study were explained. Nine of them are applied active magnetic stimuli, and other six are applied sham settings. None of them know about the settings, that is, a single-blind task.

\subsection{Tasks and procedure}

The duration of the experiment was two hours, during which ECG data was continuously recorded. The subjects were instructed to maintain a resting state for 2 minutes prior to the start of the experiment. The task was divided into five time periods. During period 1, subjects completed a 10-minute PVT, to establish an initial baseline of vigilance level. Period 2 was 60 minutes in length and induced fatigue in subjects via an N-back paradigm, so as to establish the vigilance degradation model; afterwards (Period 3), another PVT assessed vigilance. During Period 4, we used a $16 \mathrm{~Hz}$ magnetic field to stimulate subjects. During stimulation, subjects underwent a second, 30-minute $\mathrm{N}$-back task, in order to ensure that subjects did not regain vigilance as a result of the break from cognitive load during the PVT of period 3. Finally, vigilance was assessed once more via a PVT at period 5 , to determine how magnetic stimulation altered vigilance (See Fig. 1).

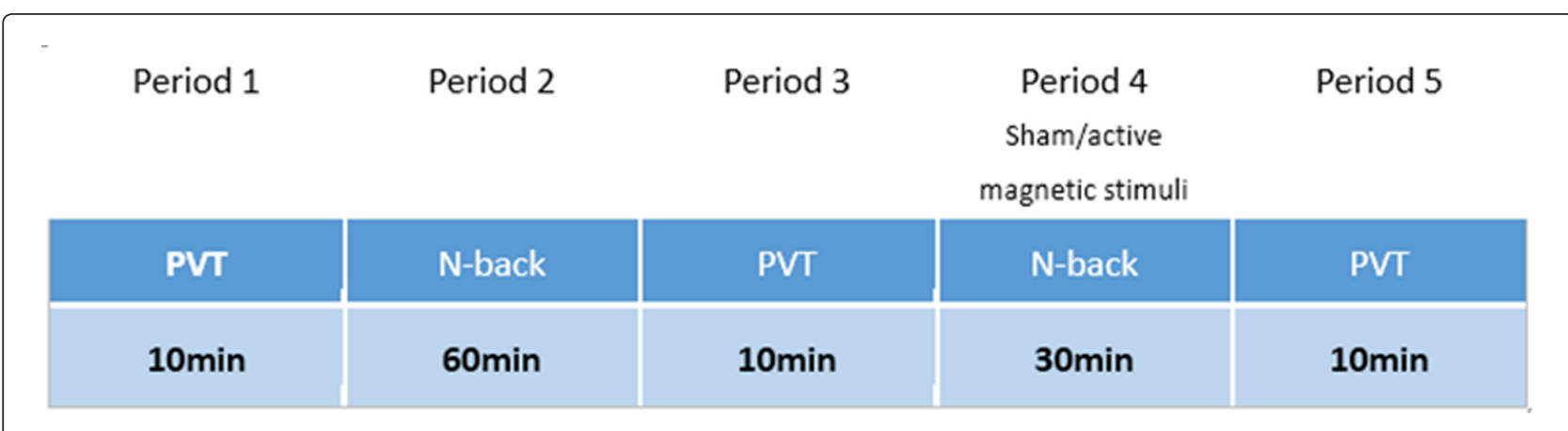

Fig. 1 Experimental process

The PVT was conducted with inter-stimulus intervals of 2 - $10 \mathrm{~s}$. Subjects were directed to focus their attention on a computer screen and to respond as quickly as possible when a displayed yellow diamond target appeared by pressing any key on the keyboard. The N-back task adjusted automatically to maintain a challenging level of difficulty, based upon the accuracy of the subjects.

Magnetic stimulation was induced via a Lowfrequency magnetic induction therapy instrument (developed by the Precision Instruments and OptoElectronics Engineering Lab, Tianjin University), generating a beta-frequency $(16 \mathrm{~Hz})$, pulsed magnetic sequence. The energy of the high frequency band rhythm of EEG, especially the beta band, is enhanced when the level of vigilance is high ${ }^{[19]}$. Therefore, we chose to use a $16 \mathrm{~Hz}$ magnetic current. Stimulation was applied continuously for 30 minutes, localized above the occipital lobe of brain. The strength was 200 Gs (i.e. gauss, or 10 - 4 tesla). (See Fig. 2)

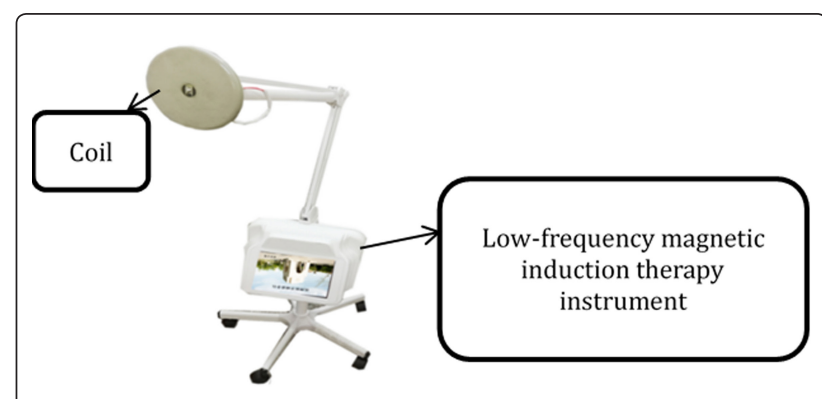

Fig. 2 Low-frequency magnetic induction therapy instrument physical map 


\subsection{ECG recording}

In this experiment, ECG signals were collected by using a BIOPAC MP150 16-channel physiological signal recording and analysis system, which can be controlled by wireless telemetry device for physiological signal measurements within 50 to 80 meters. The heart rate acquisition range was 20 250 beats per minute (bpm), and the sampling rate was $1000 \mathrm{~Hz}$.

\subsection{Time - domain analysis}

Time domain analysis method has the advantages of being simple to calculate, producing an intuitive and discrete index, and being easily comparable with other analyses of the same kind. Time domain indexes can be defined as follows: Mean $\mathrm{RR}$ constitutes the mean interval of normal sinus heartbeat; it is a reflection of the overall trend of the change of heart rate variability ${ }^{[11]}$ :

$$
\operatorname{mean} R R=\frac{\sum_{i=1}^{N} R R_{i}}{N}
$$

Root-mean-square of successive difference(RMSSD), express the speed of the change of the heart rate, between successive RR intervals:

$$
R M S S D=\sqrt{\frac{\sum_{i=1}^{M}\left(R R_{t+1}-R R_{1}\right)^{2}}{N-1}}
$$

\subsection{Frequency - domain analysis}

Frequency domain analysis converts RR intervals into a power spectrum. This enables the calculation of power spectral density (PSD), denoting how much signal falls within specific RR frequency ranges, irrespective of the place in time when RRs take place. To accomplish this, HRV signal is decomposed into various frequency bands as follows: low frequency (LF) range $(0.04 \mathrm{~Hz}-0.15$ $\mathrm{Hz}$ ), reflecting sympathetic excitability and high frequency $(\mathrm{HF})$ range $(0.15 \mathrm{~Hz}-0.4 \mathrm{~Hz})$, reflecting parasympathetic excitability.

Following this definition of frequency bands, the signal was normalized. Low-frequency power normalized value (LFn) and High-frequency power normalized value (HFn) directly reflect the changes of sympathetic and parasympathetic excitability, and were calculated as follows:

$$
\begin{aligned}
& \mathrm{LFn}=100 \mathrm{LF} /(\mathrm{TP}-\mathrm{VLF}) \\
& \mathrm{HFn}=100 \mathrm{HF} /(\mathrm{TP}-\mathrm{VLF})
\end{aligned}
$$

\section{RESULTS}

\subsection{Time Domain Results}

A paired-samples t-test was performed between the adjacent two time periods. Results revealed a significant difference in HRV components between the experimental and sham groups. Specifically, RMSSD $(p=0.007)$ (time period 4 with time period 3 ) greatly decreased for the experimental group, relative to the sham group. (See Table1.) Subjects' heart rate variability showed a downward trend when receiving magnetic stimulation. (a)

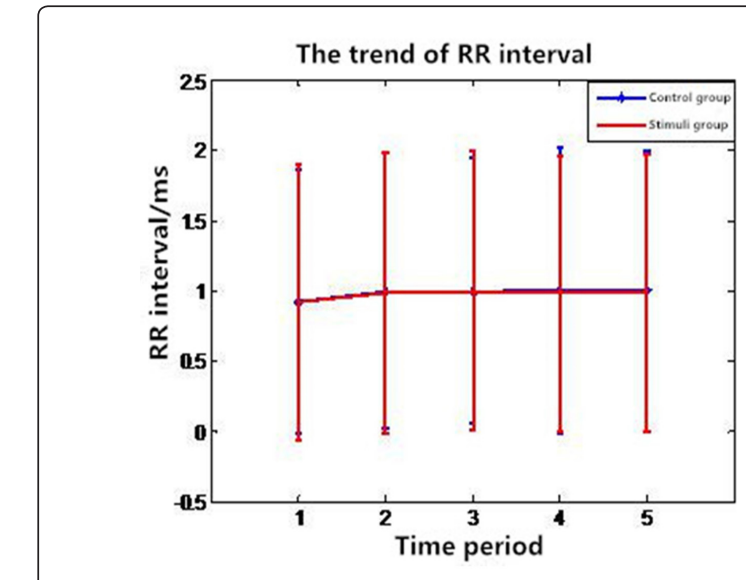

Fig. 3 Time-domain of active / sham magnetic stimuli. (a) Mean RR. (b) Root Mean Square of Successive Differences. Both (a) and (b) are the normalized results of mean RR and RMSSD in the experimental (red) and sham (blue) groups. 
Table 1. Time - Domain index Analysis of active / sham magnetic stimuli.

\begin{tabular}{|c|c|c|c|c|c|c|c|}
\hline & & & Mean RR & & & RMSSD & \\
\hline \multirow{4}{*}{$\begin{array}{l}\text { magnetic stimuli } \\
\text { group }\end{array}$} & $\begin{array}{c}\text { Time } \\
\text { period }\end{array}$ & Mean & $\begin{array}{l}\text { Standard } \\
\text { deviation }\end{array}$ & $\mathrm{Pi}$ & Mean & $\begin{array}{l}\text { Standard } \\
\text { deviation }\end{array}$ & $\mathrm{Pi}$ \\
\hline & 1 & 854.429 & 58.254 & & 28.311 & 0.261 & \\
\hline & 2 & 916.275 & 59.435 & $0.021^{*}$ & 33.015 & 0.291 & 0.069 \\
\hline & 3 & 928.092 & 58.893 & 0.291 & 34.154 & 0.308 & 0.672 \\
\hline \multirow{7}{*}{ Sham group } & 4 & 911.606 & 58.431 & 0.078 & 34.093 & 0.295 & $0.007^{*}$ \\
\hline & 5 & 915.883 & 51.888 & 0.267 & 32.914 & 0.267 & 0.067 \\
\hline & 1 & 852.027 & 59.722 & & 24.582 & 0.253 & \\
\hline & 2 & 923.971 & 62.129 & $0.025^{*}$ & 31.351 & 0.224 & $0.046^{*}$ \\
\hline & 3 & 924.941 & 60.026 & 0.224 & 31.518 & 0.284 & 0.070 \\
\hline & 4 & 927.742 & 62.892 & 0.284 & 31.801 & 0.267 & 0.069 \\
\hline & 5 & 928.753 & 63.410 & 0.264 & 31.888 & 0.264 & 0.064 \\
\hline
\end{tabular}

Note: ${ }^{*} \mathrm{P}<0.05 . \mathrm{P} 2=0.021^{*}$ is the result of period 2 and period $1 ; \mathrm{P} 3=0.291$ is the result of period 3 and period $2 ; \mathrm{P} 4=$ 0.078 is the result of period 4 and period $3 ; P 5=0.267$ is the result of period 5 and period 4 .

\subsubsection{Frequency-Domain Results}

The two groups of subjects showed differences in HRV after magnetic stimulation. The experimental group displayed reduced LFn and increased HFn. This indicates an increase of sympathetic nervous system activity and a reduction of parasympathetic nervous system activity. Summarily, HRV decreased, with the result that vigilance increased. The sham group displayed the opposite trends. However, the results of paired-samples t-test between different time periods yeilded no significant effect (magnetic stimuli group: LFn $(p=0.053)$, HFn $(p=0.081)$; sham group: LFn $(p=0.052)$ and HFn $(p=0.06)$, time period 5 with time period 4$)$.

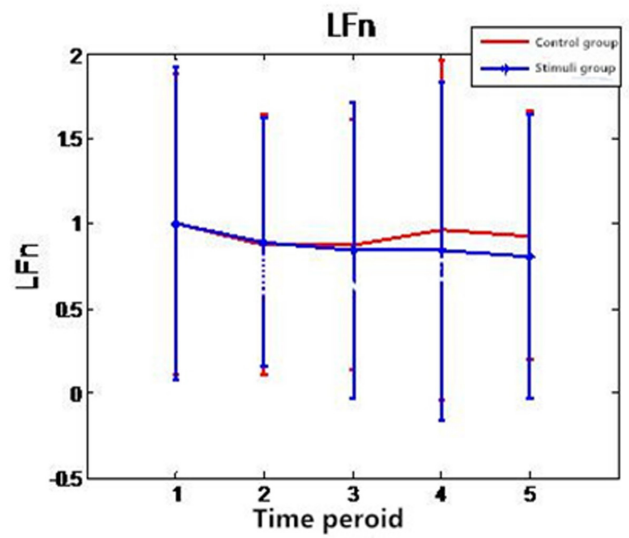

(a)

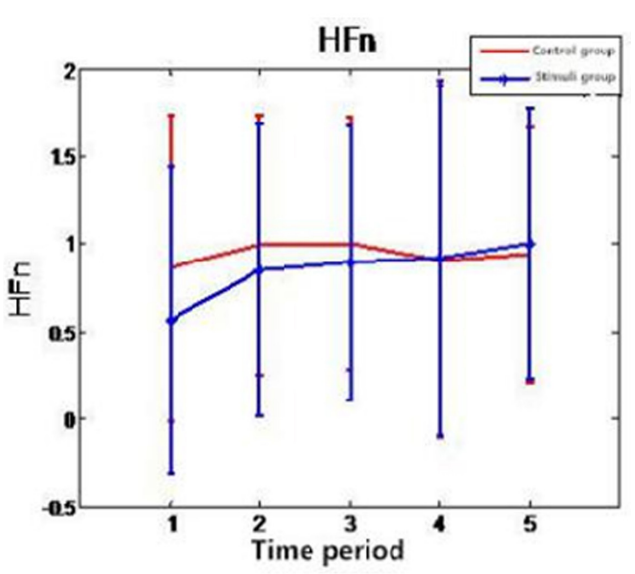

(b)

Fig. 4 Frequency domain of experimental / sham group results. (a) and (b) respectively are the normalized results of LFn and HFn in the experimental (red) and sham (blue) groups. 
Table 2. Frequency-domain index: analysis of experimental / sham group differences.

\begin{tabular}{lccccccc}
\hline & \multicolumn{3}{c}{ LFn } & \multicolumn{3}{c}{ HFn } \\
\hline $\begin{array}{l}\text { Time } \\
\text { period }\end{array}$ & Mean & $\begin{array}{c}\text { Standard } \\
\text { deviation }\end{array}$ & $\mathrm{Pi}$ & Mean & $\begin{array}{c}\text { Standard } \\
\text { deviation }\end{array}$ & $\mathrm{Pi}$ \\
magnetic & 1 & 53.929 & 11.588 & & 39.265 & 13.470 & \\
stimuli group & 2 & 47.251 & 10.116 & $0.034^{*}$ & 45.046 & 10.501 & $0.007^{* *}$ \\
& 3 & 46.972 & 9.682 & 0.08 & 45.321 & 11.134 & 0.072 \\
& 4 & 52.003 & 13.119 & 0.089 & 40.987 & 15.387 & 0.078 \\
& 5 & 50.060 & 9.639 & 0.053 & 42.607 & 9.175 & 0.081 \\
& 1 & 64.457 & 10.358 & & 13.365 & 9.529 & \\
& 2 & 57.540 & 8.236 & $0.036^{*}$ & 20.058 & 10.874 & $0.006^{* *}$ \\
& 3 & 54.494 & 9.732 & 0.073 & 21.022 & 10.264 & 0.064 \\
& 4 & 53.929 & 11.588 & 0.064 & 39.265 & 13.470 & 0.08 \\
& 5 & 52.131 & 9.357 & 0.052 & 23.456 & 9.182 & 0.06 \\
\hline
\end{tabular}

Note: ${ }^{*} \mathrm{P}<0.05 . \mathrm{P} 2=0.034^{*}$ is the result of period 2 and period $1 ; \mathrm{P} 3=0.08$ is the result of period 3 and period $2 ; \mathrm{P} 4=$ 0.089 is the result of period 4 and period $3 ; P 5=0.053$ is the result of period 5 and period 4 .

\subsection{Behavioral Results}

Response times and accuracy rates were averaged for each subject within each PVT task was recorded, after which sham and experimental groups were compared. (See Fig. 5) (The X axis represents time. Red and blue represent the experimental and sham groups, respectively.)

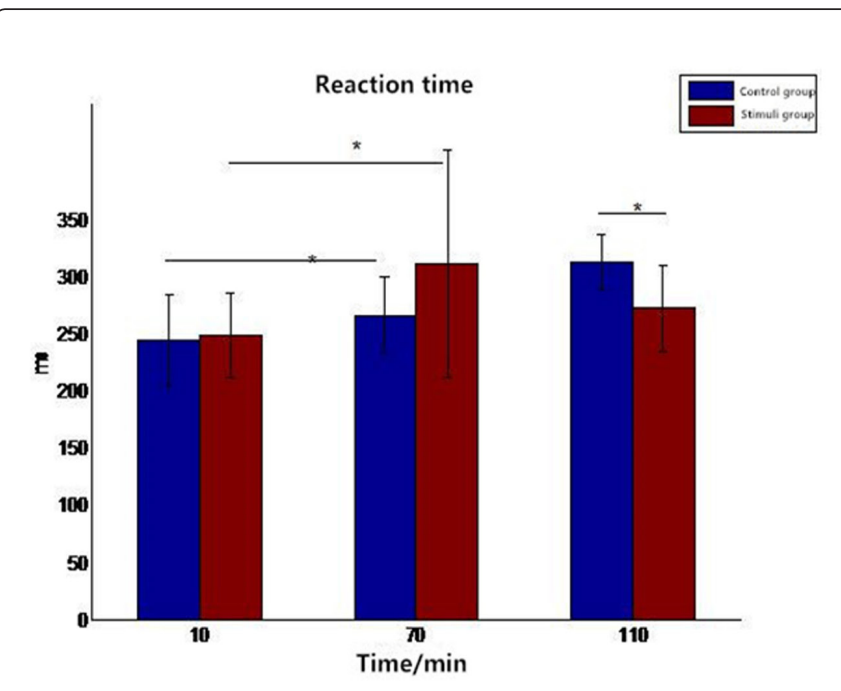

(a)

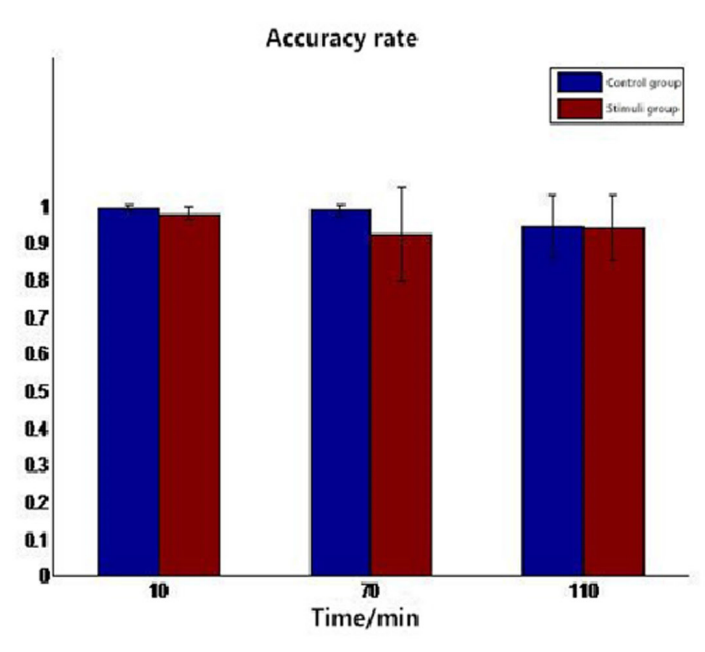

(b)

Fig. 5 Reaction time (a) and Accuracy rate (b) of PVT tasks 
As indicated by the figure, the average reaction time was decreased in the experimental group, and was increased in the sham group after magnetic stimuli. A paired-samples t-test revealed no significant difference within groups, but significant difference between groups $(p=0.0408)$. This indicates that compared with the sham group, the vigilance of experimental subjects improved.

\section{DISCUSSION}

This study examined how magnetic field pulses can be used to improve human vigilance, as indexed via HRV metrics. Comparing experimental subjects who received stimulation to sham subjects, they displayed their HRV lower frequency power, and decreased their RR, RMSSD, and HRV higher frequency power (i.e. reduced overall HRV). These markers indicate greater activation of the sympathetic nervous system and greater reduction of the parasympathetic nervous system. Thus, vigilance of the experimental subjects after cognitive depletion tasks was enhanced relative to their sham counterparts, indicating that magnetic stimulation reduced HRV and can be used to buffer vigilance degradation. This method could be useful for a number of practical situations requiring sustained vigilance to one's visual environment, such as conducting surgery or operating heavy machinery. Furthermore, the noninvasive nature of the magnetic stimulation device makes for convenient, practical delivery in realistic situations.

While this study established the ability of magnetic stimulation to ameliorate vigilance degradation, this finding could be informed in several ways. First, future studies should explore how the observed effect of buffered vigilance persists over time, with varied trial lengths. Additionally, the HRV features varied greatly during magnetic stimulation, but ceased to do so after the cessation of stimulation. Therefore, studies could apply intervallic magnetic stimuli, to attempt to keep the subjects in high vigilance conditions for longer durations in the future. Finally, though a beta-band pulse magnetic field was successfully applied to regulate the vigilance of brain, further work could also explore the relative properties of other frequency bands in order to lend context to these effects.

\section{CONCLUSION}

We believe that the present study is an important step towards improving magnetic stimulation therapies. These encouraging findings hold promise for a number of useful applications.

\section{ACKNOWLEDGEMENT}

This study is partly supported by National Natural Science Foundation of China (No. 51377120, 51007063, 31271062, 81222021, 61172008 and 81171423), Natural Science Foundation of Tianjin (No. 13JCQNJC13900), National Key Technology R\&D Program of the Ministry of Science and Technology of China (No. 2012BAI34B02) and Program for New Century Excellent Talents in University of the Ministry of Education of China (No. NCET-10-0618).

\section{CONFLICT OF INTEREST}

The authors claims no conflict of interest.

\section{REFERENCES}

1. Lim J, Dinges DF. Sleep deprivation and vigilant attention. Ann N Y Acad Sci. 2008 May; 1129: 304-322.

2. Davis $M$, Whalen PJ. The amygdala: vigilance and emotion. Mol Psychiatry. 2001 Jan; 6(1): 1334.

3. Mander BA, Santhanam S, Saletin JM, Walker MP. Wake deterioration and sleep restoration of human learning. Curr Biol. 2011 Mar; 21(5): R182-R184.

4. Munde VS,Vlaskamp C, Ruijssenaars AJJM, Nakken $\mathrm{H}$. Alertness in individuals with profound intellectual and multiple disabilities: a literature review. Research in Developmental Disabilities. 2009 May; 30(3): 462-480.

5. Robertson IH, Manly T, Andrade J, Baddeley BT, Yiend J. Oops!: performance correlates of everyday attentional failures in traumatic brain injured and normal subjects. Neuropsychologia. 1997 May; 35(6): 747-758.

6. Langner R, Eickhoff SB. Sustaining attention to simple tasks: a meta-analytic review of the neural mechanisms of vigilant attention. Psych Bull. 2013 Nov; 139(4): 870-900.

7. Chua EC, Tan WQ, Yeo SC. Heart Rate Variability Can Be Used to Estimate Sleepinessrelated Decrements in Psychomotor Vigilance 
during Total Sleep Deprivation. Sleep. 2012 Mar; 35(3): 325-334.

8. Kamath MV, Fallen EL. Power spectral analysis of heart rate variability: a noninvasive signature of cardiac autonomic function. Crit Rev Biomed Eng. 1993 Dec; 21(3): 245-311.

9. Martinmäki K, Rusko H, Saalasti S. Ability of short-time Fourier transform method to detect transient changes in vagal effects on hearts: a pharmacological blocking study. Am JPhysiol. 2006 Jun; 290(6): 2582-2589.

10. Sun $Y, Y u X B$. An innovative nonintrusive driver assistance system for vital signal monitoring. IEEE J Biomed Health Inform. 2014 Feb; 18(6): 1932-1939.

11. Porges SW, Raskin DC. Respiratory and heart rate components of attention. J Exp Psychol. 1969 Sep; 81(81): 497-503.

12. Riemersma AR, Sanders C. Performance determent during prolonged night driving. New York: Flenum Press. 1977; 41-58.

13. Dinges DF, Powell JW. Microcomputer analyses of performance on a portable, simple visual RT task during sustained operations. Behav Res Methods. 1985 Nov; 17(6): 652-655.

14. Thomann J, Baumann CR, Landolt HP, Werth
E. Psychomotor vigilance task demonstrates impaired vigilance in disorders with excessive daytime sleepiness. J Clin Sleep Med. 2014; 10(9): 1019-1024.

15. Thomas AW, Drost DJ, Prato FS. Human subjects exposed to a specific pulsed (200 microT) magnetic field: effects on normal standing balance. Neurosci Lett. 2008; 297 : 121-124.

16. Cook CM, Thomas AW, Keenliside L, Prato FS. Resting EEG effects during exposure to a pulsed ELF magnetic field. Bioelectromagnetics. 2005; 26(26): 367-76.

17. Mehran Y Z, Firoozabadi M, Rostami R. Analysis of EEG rhythms under local sinusoidal ELF magnetic field exposure: An approach to neurofeedback enhancement on attention performance. J Biomed Sci Eng. 2013; 6(10): 947-953.

18. Shi LC,Jiao YY, Lu BL.Differential Entropy Feature for EEG-based Vigilance Estimation. Conf Proc IEEE Eng Med Biol Soc. 2013; 2013: 6627-6630.

19. Steriade M. Grouping of brain rhythms in corticothalamic systems. Neuroscience. 2006; 137(4): 1087-1106. 